\title{
A Theoretical Consideration of the Ballistic Response of Continuous Graphene Membranes
}

\author{
Eric D. Wetzel*, Radhakrishnan Balu, and Todd D. Beaudet \\ U.S. Army Research Laboratory
}

Weapons and Materials Research Directorate and Computational and Information Sciences

Directorate

Aberdeen Proving Ground, MD 21005

*eric.d.wetzel2.civ@mail.mil, 410-306-0851

\author{
Submitted to: \\ Journal of the Mechanics and Physics of Solids \\ Submission date: 22 January 2015 \\ Revision date: 30 April 2015
}

\begin{abstract}
The remarkable properties of graphene, including unusually high mechanical strength and stiffness, have been well-documented. In this paper, we combine an analytical solution for ballistic impact into a thin isotropic membrane, with ab initio density functional theory calculations for graphene under uniaxial tension, to predict the penetration resistance of multilayer graphene membranes. The calculations show that continuous graphene membranes could enable ballistic barriers of extraordinary performance, enabling resistance to penetration at masses up to $100 \times$ lighter than existing state-of-the-art barrier materials. The very high elastic wave speed and strain energy to failure are the major drivers of this increase in performance. However, the in-plane mechanical isotropy of graphene, as compared to conventional orthotropic
\end{abstract}


woven textiles, also contributes significantly to the efficiency of graphene as a barrier material. This result suggests that, for barrier applications, isotropic membranes composed of covalently bonded two-dimensional molecular networks could provide distinct advantages over fiber-based textiles derived from linear polymers.

Keywords: graphene, ballistics, armor, impact, textiles

\section{Background}

Ballistic barriers for body armor, turbine blade confinement, or space debris shields are important technological structures for which the common goal is to resist penetration from a high velocity projectile, usually while minimizing weight and/or bulk. In many cases, textiles woven from high performance yarns such as aramids (for example DuPont ${ }^{\mathrm{TM}}$ Kevlar $^{\circledR}$ fibers) or ultrahigh molecular weight polyethylene (UHMWPE) (for example Honeywell Spectra ${ }^{\circledR}$ fibers) are major components in these barrier materials, primarily because they have among the highest densitynormalized stiffness and strength of commercially available materials. In other specialty armors, not of interest to the present discussion, hard ceramic strike faces are also included to break up the projectile and consume energy during the comminuted flow.

The recent emergence of graphene as a material of extraordinary strength and stiffness [1, 2] suggests that it may provide unprecedented capabilities as a ballistic barrier material. Indeed, recent experimental results [3] are consistent with this expectation. In this paper, a theoretical estimation of the potential performance of graphene as a ballistic barrier is presented. First, analytical impact theory is used to establish scaling parameters for ballistic penetration response, and highlight the fundamental difference in response for isotropic membranes such as graphene 
versus orthotropic, woven textiles. The in-plane uniaxial stress response for a graphene membrane is then generated via ab initio density functional theory, and applied to estimate ballistic scaling parameters for graphene and predict the behavior of graphene relative to conventional ballistic barrier materials. In addition, to eliminate some of the uncertainty associated with estimating graphene performance using a linear elastic, small deformation theory, predictions are also provided for a "sub-graphene" notional material with elastic properties identical to graphene, but exhibiting small strains to failure.

\section{Isotropic versus Orthotropic Ballistic Barriers}

The mechanics of ballistic impact are complex, but the case of normal impact into a thin isotropic membrane can nominally be described as follows [4]. Upon initial contact, a momentum exchange between the projectile and the barrier leads to a rapid partial deceleration of the projectile. A radial in-plane tensile wave then emanates from the impact point at a speed comparable to the elastic wave speed in the material, pulling barrier material towards the projectile. Simultaneously, the projectile is travelling normal to the barrier, creating a transverse deformation cone which continuously decelerates the projectile. Radial tensile loads build in the vicinity of the projectile and, if local stresses exceed failure conditions for the barrier, perforation occurs. If, instead, the projectile is completely decelerated prior to penetration, the projectile is stopped. In this model of barrier impact, the primary loads on the barrier are in-plane tension and, therefore, it is these properties that are the major determinant of ballistic response.

The ballistic response of most barriers is probabilistic; that is, a given set of ballistic conditions (consistent armor composition, armor areal density, mechanical boundary conditions, and projectile characteristics) can result in either a penetrative or non-penetrative event. The 


\section{Wetzel et al., "A Theoretical Consideration of the Ballistic Response of Continuous Graphene Membranes"}

probability of penetration tends to increase as impact velocity increases, with penetration being highly unlikely at very low velocities and nearly certain to occur at very high velocities. The first-order parameter that is typically measured and reported to represent the characteristic ballistic limit of a particular barrier is the velocity at which the probability of penetration is $50 \%$, referred to as the $V_{50}$ velocity [5]. For analysis purposes, this velocity represents, on average, the velocity at which the projectile is just barely stopped by the barrier. Said differently, the $V_{50}$ represents, in an average sense, the condition where the initial kinetic energy of the projectile is exactly balanced by the available deformation and failure energy within the interacting volume of the barrier material.

Using dimensional analysis and empirical fitting to experimental ballistic data, Cunniff [6] showed that the limiting penetration velocity for a homogeneous (all layers composed of identical materials) textile barrier scales with the material parameter

$$
\Lambda_{y}=\left(\bar{U}_{y} \cdot c_{y}\right)^{1 / 3}
$$

where $\bar{U}_{y}$ is the mass-normalized tensile strain energy to failure $(\mathrm{J} / \mathrm{g})$ of the yarns in the textile, and $c_{y}$ is the longitudinal elastic wavespeed in the yarns, given by

$$
c_{y}=\sqrt{\frac{E_{y}}{\rho}} .
$$

Here, $E_{y}$ is the longitudinal elastic modulus of the yarns. Using similar scaling arguments, Cunniff also hypothesized that the performance of a barrier material should be driven by the mass ratio parameter $\Gamma$, defined as

$$
\Gamma=\Theta \frac{\rho h}{m_{p} / A_{p}}
$$


where $m_{p}$ is the projectile mass, $A_{p}$ is the projectile presented area, $\rho$ is the barrier density, and $h$ is the barrier thickness. $\Theta$ is a factor to account for effects such as obliquity and projectile blunting, with a typical value of 1.5-1.8 required for good agreement between theory and experimental data [4]. To demonstrate the utility of these scaling parameters, Cunniff showed that ballistic data for a variety of aramid and nylon textiles and textile composites could be collapsed to a single empirical function for $V_{50} / \Lambda_{y}$ as a function of $\Gamma$. The same empirical function tended to overpredict the ballistic performance for UHMWPE textiles and composites reinforced with UHMWPE, carbon fiber, and glass fiber.

Following this scaling study, Porwal and Phoenix [4] derived an analytical solution for the impact of an infinite, linear elastic, thin isotropic membrane by a cylindrical projectile. The derivation assumes a self-similar deformation cone shape, neglects Poisson's ratio effects (resulting in very small errors for materials with Poisson's ratio less than 0.3 , a condition predicted to be met for graphene by most simulations to date [7]), and eliminates many higher order terms (insignificant in value for most practical ballistic conditions) in order to produce a closed-form analytical solution for the strain and displacement history of the impacted membrane. This analysis predicts that the membrane limiting velocity ( $\left.V_{50}^{\text {membrane }}\right)$ should be proportional to

$$
V_{50}^{\text {membrane }}=\sqrt{2}(1+\Gamma) c_{m}\left(\varepsilon_{m} / K_{m}\right)^{3 / 4}
$$

where $c_{m}$ is the linear wavespeed in the membrane, $\varepsilon_{m}$ is the maximum strain to failure in the membrane, $K_{m}$ is a function that depends on both $\Gamma$ and $\varepsilon_{m}$. Since the solution is derived for a linear elastic material, this relation can be recast as

$$
\frac{V_{50}^{\text {membrane }}}{\Lambda_{m}}=2^{5 / 6}(1+\Gamma) \varepsilon_{m}^{1 / 12} K_{m}^{-3 / 4}
$$


where

$$
\Lambda_{m}=\left(\frac{\sigma_{m} \varepsilon_{m}}{2 \rho} \cdot c_{m}\right)^{1 / 3}=\left(\frac{E_{m} \varepsilon_{m}^{2}}{2 \rho} \cdot c_{m}\right)^{1 / 3}=c_{m}\left(\frac{\varepsilon_{m}^{2}}{2}\right)^{1 / 3}
$$

and $E_{m}$ and $\sigma_{m}$ are the tensile elastic modulus and failure stress in the membrane, respectively. Equation (5) shows that $\Lambda_{m}$ alone is not rigorously sufficient to capture the effects of material properties on $V_{50}$ scaling, since $\varepsilon_{m}$ appears on the right-hand-side (RHS) of this equation (including within the $K_{m}$ function). However, it can be shown that $\Lambda_{m}$ varies more strongly with $\varepsilon_{m}$ compared to the variation of the RHS of Eqn. (5) with respect to $\varepsilon_{m}$; for example, for a value of $\Gamma=0.2$ (representative of a $1 \mathrm{gr}$ steel projectile impacting a typical 30-layer Kevlar target) as $\varepsilon_{m}$ varies from 0.01 to $0.2, \Lambda_{m}$ varies by a factor of 7 while the RHS of Eqn. (5) varies only by a factor of 1.9. Therefore Porwal and Phoenix argue that it is reasonable, although not exact, to use $\Lambda_{m}$ as a material scaling factor for the prediction of $V_{50}^{\text {membrane }}$ performance.

To apply this solution for isotropic membranes to plain-woven (orthogonal yarn) textiles, Porwal and Phoenix modify their solution by accounting for the fact that only half of the yarns in the fabric contribute to loading in each principal direction. The final solution for predicted ballistic limit for a textile $\left(V_{50}^{\text {textile }}\right)$ is

$$
\frac{V_{50}^{\text {textile }}}{\Lambda_{y}}=2^{1 / 3}(1+\Gamma) \varepsilon_{y}^{1 / 12} K_{y}^{-3 / 4}
$$

where $\Lambda_{y}$ and $K_{y}$ are functions identical to $\Lambda_{m}$ and $K_{m}$, but are calculated using elastic yarn properties $E_{y}, \varepsilon_{y}$ (strain to failure), and $\sigma_{y}$ (failure stress). Comparing this equation to the relationship for the $V_{50}$ of an isotropic elastic membrane (Eqn. 5), under conditions of identical yarn and membrane tensile properties we find that

$$
V_{50}^{\text {membrane }}=\sqrt{2} \cdot V_{50}^{\text {textile }}
$$


This relationship provides our first crucial result: comparing an isotropic membrane to a woven textile of identical mass, if the textile yarns have identical tensile properties to the isotropic membrane, the critical penetration velocity for the membrane will be approximately $40 \%$ higher than the woven textile. Despite this advantage, conventional isotropic membranes, such as metal foils and polymer films with randomly oriented linear polymers, do not have sufficient densitynormalized mechanical strength and stiffness to compete with state-of-the-art fibers composed of highly aligned linear polymers. The emergence of graphene and other two-dimensional polymers presents an opportunity to reconsider isotropic membranes.

\section{Mechanical Response of Graphene}

To evaluate the potential of graphene as a ballistic barrier, its representative mechanical properties are required. We can limit our consideration to uniaxial tension, as the tensile radial stresses during ballistic impact far exceed the slightly compressive circumferential (hoop) stresses (which, in practicality, are most likely accommodated via out-of-plane buckling rather than true compression) [4]. Prior experimental [1] and computational studies [8,9] have shown that graphene at high strains obeys a non-linear elastic response which, under uniaxial tension, can be expressed phenomenologically as

$$
\sigma=E_{m} \varepsilon+D_{m} \varepsilon^{2}
$$

where $\sigma$ is the tensile stress (first Piola-Kirchoff stress), $\varepsilon$ is the tensile strain (change in length normalized by original length), $E_{m}$ is the linear Young's modulus, and $D_{m}$ is the non-linear elastic modulus. Since a graphene monolayer does not have a well-defined thickness, stress and moduli are expressed as two-dimensional values with units of $\mathrm{N} / \mathrm{m}$. For a negative $D_{m}$ value, the 
maximum stress occurs at $\sigma_{m}=-E_{m}^{2} /\left(4 D_{m}\right)$ and $\varepsilon_{m}=-E_{m} /\left(2 D_{m}\right)$. Integrating Eqn. (9) with respect to strain gives the strain energy density $U$ of the material during uniaxial tension:

$$
U=\frac{1}{2} E_{m} \varepsilon^{2}+\frac{1}{3} D_{m} \varepsilon^{3}
$$

The strain energy at failure $\left(\varepsilon=\varepsilon_{m}\right)$ is therefore $U_{m}=4 \sigma_{m}^{2} /\left(3 E_{m}\right)$ where, if two-dimensional moduli are used for $E_{m}$ and $D_{m}$, then $U_{m}$ is an area-normalized energy $\left(\mathrm{J} / \mathrm{m}^{2}\right)$.

Values of $E_{m}$ and $D_{m}$ for graphene have been reported, primarily calculated via molecular dynamics [8-10] but also supported by at least one experimental data set [1] (Table 1). We complement this data by performing ab initio Kohn-Sham density functional theory (DFT) based computations using the Gaussian and plane wave-method code QUICKSTEP as implemented in CP2K [11]. The calculations use the generalized gradient approximation (GGA) exchange-correlation functional BLYP due to Becke [12] and Lee, Yang, Parr [13]. The 1s $\mathrm{s}^{2}$ carbon core states are accounted for by Goedecker, Teter, and Hutter (GTH) pseudopotential [14, 15] optimized for BLYP by Krack [16]. A Gaussian basis set with double-zeta and valence polarization functions fit for BLYP/GTH was used to describe the single-body orbitals. The density was described by plane wave basis with 350 Ry cutoff. We treat the Gamma point of the Brillouin zone using a super cell $(4 \times 4 \times 1$ unit cell) with 32 atoms and graphene layers spaced by $15 \AA$ A. Dispersion interactions are accounted for using the DFT-D method with D3 parameterization as implemented in $\mathrm{CP} 2 \mathrm{~K}[17,18]$. Total energies, energy gradients and lateral stresses were converged to $1.0 \times 10^{-6}$ Hartree, $4.5 \times 10^{-4}$ Hartree/Bohr, and 100 bar respectively throughout. Calculations were performed for uniaxial tension in both the zigzag (second nearest neighbor) and armchair (nearest neighbor) directions [8], at strain increments of 0.5\% up to peak strains of $27 \%$ and $25 \%$ strain, respectively. The CP2K framework accepts finite-strain initial 
conditions, therefore the prediction of uniaxial stress response required the use of an external script. Using the stress-free equilibrium structure as an initial condition, a uniaxial strain of $0.5 \%$ was applied in the direction of interest. The lateral strain was then relaxed slightly, and a new solution calculated, resulting in reduced lateral stress. This process of transverse relaxation was performed iteratively via the external script, until the lateral stress was reduced to zero within the 100 bar convergence limit. This process was repeated in increments of $0.5 \%$ strain to generate the overall material response curves in the armchair and zigzag directions. The resulting lattice energy, normalized by initial unit cell area, and stress responses are shown in Fig. 1. The energy data was fitted according to Eqn. (10) in both armchair and zigzag directions simultaneously, producing a single $E_{m}$ value and separate armchair and zigzag $D_{m}$ values. The results are given in Table 1 , with a single $D_{m}$ reported as the orientationally averaged value (equal to the simple average of zigzag and armchair directions [8]). Values of $E_{m}=329 \mathrm{~N} / \mathrm{m}$ and $D_{m}=-691 \mathrm{~N} / \mathrm{m}$, calculated as the average of all reported values from Table 1, are used for subsequent quantitative estimations of graphene mechanical response.

The two-dimensional (areal) density of monolayer graphene can be estimated to be $7.42 \times$ $10^{-7} \mathrm{~kg} / \mathrm{m}^{2}$, based on hexagonal symmetry and a bond length of $0.144 \mathrm{~nm}$ (the equilibrium configuration from $\mathrm{CP} 2 \mathrm{~K}$, very close to the value of $0.142 \mathrm{~nm}$ reported for single crystal graphite [19]). Combining this areal density with the elastic modulus from Table 1, Eqn. (2) gives a lowstrain wavespeed for graphene of $21.0 \mathrm{~km} / \mathrm{s}$. Normalizing the per-area energy to failure from Table 1 by the areal density gives a mass-normalized energy to failure of $8350 \mathrm{~J} / \mathrm{g}$. 


\section{Theoretical Ballistic Predictions for Graphene}

Using the average wavespeed and deformation energy at failure from Table 1, the ballistic parameter of Eqn. (6) has a value of $\Lambda_{m}=5600 \mathrm{~m} / \mathrm{s}$ for graphene. This value is compared to other engineering materials in Table 2. Materials above the dashed line are textiles, while materials below the dashed line are isotropic membranes. Also tabulated are ballistic limits normalized by the ballistic limit of a Kevlar membrane of comparable mass. These ratios are calculated by assuming that $V_{50}$ is proportional to $\Lambda$, and that isotropic membranes will have a ballistic limit that is higher than woven textiles of comparable mechanical properties by a factor of $\sqrt{2}$ per Eqn. (8). The results show that most engineering textiles are expected to provide comparable ballistic response, with emerging materials such as spider silk (the value given is the highest strain energy density reported for spider silk, based on a unique species [20]) and carbon nanotube (CNT)-based yarns [22] providing opportunities for more significant performance enhancements. Conventional metallic and polymer isotropic membranes perform similar to, or worse than, Kevlar textile membranes. In comparison, graphene, with isotropic properties and relatively high wave speed and strain energy density, is expected to produce a remarkable $12 \times$ increase in ballistic limit.

Equation (5) was derived for a linear elastic material at low strains. The application of this theory to graphene, with non-linear elastic behavior and high strain-to-failure, results in predictions with considerable uncertainty. Therefore it is instructive to also consider a "subgraphene" membrane, with an $E_{m}$ equal to graphene but assumed to exhibit linear elastic behavior to a failure strain of only 3.3\% (matched to Kevlar), given in Table 2. The simulations confirm that graphene exhibits linear response at low strains (Fig. 1), and by matching the peak strain to that of Kevlar the weaker strain dependencies in the RHS of Eqn. (5) are rigorously 
eliminated from the $V_{50}$ ratio calculation. Furthermore, since it may not be possible to produce single-crystal graphene at engineering scale, the sub-graphene can also represent an imperfect graphene membrane whose strength is compromised by flaws such as vacancies and grain boundaries. In spite of the drastically reduced strain to failure, this material is still expected to possess a $V_{50}$ value over $3 \times$ higher than a Kevlar barrier of comparable mass.

It is also critical to determine the mass of graphene membrane necessary to match the ballistic limit of a given Kevlar barrier, i.e. the potential weight savings. The barrier areal density is captured by the barrier-to-projectile mass ratio $\Gamma$, but since $\Gamma$ is also embedded within $K$ one can not easily separate mass effects from Eqns. (5) and (7). As a simple approximation, the projectile kinetic energy scales with the square of its velocity, therefore the square of the $V_{50}$ ratio given in Table 2 is a crude metric of the relative failure energy density of the barrier, and one would therefore expect that

$$
\frac{\Gamma_{m}}{\Gamma_{\text {Kevlar }}} \approx \frac{1}{2}\left(\frac{\Lambda_{\text {Kevlar }}}{\Lambda_{m}}\right)^{2}
$$

where the factor of $1 / 2$ is necessary per Eqn. (8) since we are comparing a woven textile to an isotropic membrane. This relation predicts that a sub-graphene membrane would be roughly $8 \%$ the mass of a Kevlar barrier with comparable $V_{50}$, while a graphene membrane would be approximately $0.7 \%$ of the Kevlar barrier mass. To provide higher confidence in this trend, we implement the full solutions of Eqn. (5) for sub-graphene and graphene, and Eqn. (7) for Kevlar (see [4] for full expansion of $K$ in terms of $\varepsilon$ and $\Gamma$ ). Calculations are performed using the properties of Table 2, over a range of $\Gamma$ for each material that gives $V_{50}$ values from 100-1000 $\mathrm{m} / \mathrm{s}$ (typical of barrier applications such as body armor) (Fig. 2a). This data is then used to calculate mass ratios for graphene and sub-graphene relative to Kevlar at each $V_{50}$. Interestingly, 
these mass ratios show only a weak dependence on $\Gamma$ (via $K$ in Eqns. (5) and (7)); the average mass ratio values are $12 \%$ for sub-graphene and $0.5 \%$ for graphene over the full range of $\Gamma$. Empirically we find that the analytical data is well-fit (Fig. 2) by the relation

$$
\frac{V_{50}}{\Lambda} \approx \frac{1}{\alpha} \cdot 2^{13 / 4} \varepsilon^{1 / 3} \Gamma^{3 / 5}
$$

where $\alpha=1$ for isotropic membranes and $\alpha=\sqrt{2}$ for textiles per Eqn. (8). Using this empirical relationship, the mass ratio is then predicted to be

$$
\frac{\Gamma_{m}}{\Gamma_{\text {Kevlar }}} \approx \frac{1}{2^{5 / 6}}\left(\frac{\Lambda_{\text {Kevlar }}}{\Lambda_{m}}\right)^{5 / 3}\left(\frac{\varepsilon_{\text {Kevlar }}}{\varepsilon_{m}}\right)^{5 / 9}
$$

which gives mass ratios (relative to Kevlar) of $12 \%$ for sub-graphene and $0.6 \%$ for graphene. Taken together, these calculations predict that a sub-graphene barrier would be roughly $10 \times$ lighter than a comparable Kevlar barrier, while a graphene barrier would be over $100 \times$ lighter.

\section{Discussion and Conclusions}

These theoretical predictions have a number of caveats. First, as mentioned above, the ballistic penetration theory was derived under the assumption of low strains and linear elasticity, while the full graphene response is non-linear elastic and fails at relatively high strains; improved predictions require a more general theory that is not presently available. (However, it should also be noted that the basic material parameters of the model - wavespeed and mass-normalized energy to failure - are reasonably well-characterized for graphene, and therefore the basic scaling arguments presented herein should hold to order-of-magnitude accuracy.) Secondly, the theory assumes that membrane failure is dominated by in-plane tension, and that other local loads such as transverse compression and shear do not trigger premature failure. This assumption often holds true for high performance fibers like Kevlar and UHMWPE that are unusually adept at 
maintaining tensile strength in the presence of transverse loading [24-26]. Little is known about graphene failure under multi-axial loading. Similarly, the model does not take into consideration flaw effects, grain boundaries, and crack growth. Simulations of failure in graphene predict significant strength reductions due to grain boundaries [27-29] and atomic vacancies [30]. While experiments to directly measure fracture toughness have not achieved consensus [31-32], other experiments have demonstrated that the strength and stiffness of polycrystalline graphene films as measured via center-loading of a suspended membrane can be comparable to values for single crystal graphene if best-practices synthesis and transfer steps are implemented [3]. Finally, this analysis derives mechanical properties from single layer graphene simulations, while the ballistic predictions are meant to represent many layers of graphene loaded simultaneously. Simulations and experiments are needed to better understand how loads are shared between graphene layers during ballistic impact. We also note that the theory requires membrane behavior with negligible bending stiffness, while millions of graphene layers (e.g. crystalline graphite) behaves like a rigid plate. A flexible graphene ballistic barrier would need to be composed of many thin, individual graphitic sub-membranes, each composed of hundreds or perhaps thousands of layers of graphene but mechanically distinct so that the assembly remains flexible, similar in form to a stack of high strength films or foils.

Considering these caveats, it is useful to compare our theoretical predictions relative to very recent "microballistic" experiments on many-layer graphene (MLG) membranes [3]. In these experiments, edge-supported, unbacked HOPG membranes of 10-100 nm thickness (30300 layers of graphene) are penetrated by $3.7-\mu \mathrm{m}$-diameter glass spheres with an impact velocity of 600 or $900 \mathrm{~m} / \mathrm{s}$. The results show that the per-mass energy absorbed by the MLG membranes is 5-10 $\times$ higher than those of gold films and polymer films. Furthermore, using scaling 
arguments to compare these results to macroscopic ballistic data, the energy absorption in the MLG membranes is approximately $50 \%$ higher than that of Kevlar composite membranes. Although this improved performance relative to Kevlar quite impressively demonstrates graphene's efficiency as a ballistic barrier, the present theory predicts that the energy absorption has the potential to be $10-100 \times$ higher than Kevlar. Significant crack propagation away from the impact site could be an indication that a more detailed treatment of flaw effects and fracture mechanics is needed to explain the experimental data set relative to theory. It should also be noted that the theory is derived for an infinitely large membrane, whereas the experimental membranes are finite in size. Considering the high in-plane wave speed for graphene, it is likely that significant stress wave reflections and interactions exist in the experiments, which could generate stress concentrations that trigger premature failure.

We also note a number of secondary advantages of isotropic membranes compared to woven textiles. The strength of yarns can be significantly degraded (up to 20\%) during the weaving process [33], so the final barrier strength of a textile may lag behind its theoretical potential. Isotropic membranes, in contrast, could be stacked to form ballistic barrier, avoiding the mechanical degradation associated with weaving. Yarns are not closely held together, therefore "windowing" processes may occur that allow sharp-tipped projectiles or stabbing threats to push aside yarns and penetrate without significant fiber failure [34]; such a mechanism would not be operable in an isotropic membrane. Woven textiles also possess crimp (yarn undulations) that straighten during impact, which can exaggerate the transverse deflection cone exhibited by the barrier; planar membranes have no crimp, and therefore should be capable of stopping projectiles at much lower backface deflection values, reducing secondary damage to supporting materials or minimizing blunt trauma to a body armor wearer. Roylance et al. [35] 
also speculated that yarn cross-overs trigger local longitudinal stress wave reflections that can induce premature barrier failure.

In summary, we have shown that multi-layer stacks of continuous graphene membrane could provide a revolutionary advance in ballistic barriers. In fact, graphene is only one example of the broad field of two-dimensional polymers [36-44], many of which would be expected to exhibit nominally isotropic high strength and stiffness. Other two-dimensional materials such as $\mathrm{BN}$ [45] and $\mathrm{MoS}_{2}$ [46] are also predicted to provide excellent in-plane mechanical properties although at values that are somewhat lower than graphene. The major challenge is to develop techniques to fabricate these materials at sufficient scale for technological use. We note that, even if the $100 \times$ mass savings for graphene versus Kevlar is realized, over 50,000 layers of graphene would be needed to create a barrier comparable to a $4 \mathrm{~kg} / \mathrm{m}^{2}$ Kevlar body armor. The continuous nature of the membrane is key, therefore we would expect that using finite graphene flakes (smaller in size than the projectile diameter, e.g. $10 \mathrm{~mm}$ or smaller) as reinforcement in a polymer will not provide the necessary performance. Scalable techniques for growing large sheets, or stitching together finite sheets, at a massive scale are needed. One should also consider that carbon nanotube yarns can, in theory, produce equivalent mechanical properties to graphene. However, the theoretical arguments of the present study suggest that a continuous graphene membrane would considerably outperform ( $40 \%$ higher $\left.V_{50}\right)$ an equivalent mass carbon nanotube textile.

\section{Acknowledgements}

This research was supported in part by an appointment to the Postgraduate Research Participation Program at the U.S. Army Research Laboratory administered by the Oak Ridge 
Institute for Science and Education through an interagency agreement between the U.S.

Department of Energy and ARL. This work was made possible by a grant from the ARL DoD Supercomputing Resource Center (DSRC) modernization program.

\section{References}

1. C. Lee, X. Wei, J. W. Kysar, and J. Hone. "Measurement of the elastic properties and intrinsic strength of monolayer graphene." Science. v321 p385-388. 2008.

2. G.-H. Lee, R. C. Cooper, S. J. An, S. Lee, A. van der Zande, N. Petrone, A. G. Hammerberg, C. Lee, B. Crawford, W. Oliver, J. W. Kysar, and J. Hone. "High strength chemical-vapordeposited graphene and grain boundaries." Science. v340 p1073-1076. 2013.

3. J.-H. Lee, P. E. Loya, J. Lou, and E. L. Thomas. "Dynamic mechanical behavior of multilayer graphene via supersonic projectile penetration." Science. v346 n6213 p1092-1096. 2014.

4. S. L. Phoenix and P. K. Porwal. "A new membrane model for the ballistic impact response and V50 performance of multi-ply fibrous systems." Int. J. Solids Struct. v40 p6723-6765. 2003.

5. Q. J. Nottingham, J. B. Birch, and B. A. Bodt. "Local logistic regression: An application to Army penetration data." J. Statist. Comput. Simul. v66 p35-50. 2000.

6. P. M. Cunniff. "Dimensionless parameters for optimization of textile-based body armor systems." Proc. of 18th Int. Symp. on Ballistics. San Antonio, TX. November 15-19, 1999. p1303-1310.

7. G. Cao. "Atomistic studies of mechanical properties of graphene." Polymers. v6 p24042432. 2014. 
8. E. Cadelano, P. L. Palla, S. Giordano, and L. Colombo. "Nonlinear elasticity of monolayer graphene." Phys. Rev. Lett. v102 n235502. 2009.

9. G. Kalosakas, N. N. Lathiotakis, C. Galiotis, and K. Papagelis. "In-plane force fields and elastic properties of graphene." J. Appl. Physics. v113 n134307. 2013.

10.F. Liu, P. Ming, and J. Li. "Ab initio calculation of ideal strength and phonon instability of graphene under tension." Phys. Rev. B v76 n064120. 2007.

11.J. VandeVondele, M. Krack, F. Mohamed, M. Parrinello, T. Chassaing, and J. Hutter. "QUICKSTEP: Fast and accurate density functional calculations using a mixed Gaussian and plane waves approach." Comp. Phys. Commn. v167 p103. 2005.

12.A. D. Becke. "Density-functional exchange-energy approximation with correct asymptotic behavior.” Phys. Rev. A. v38 p3098-3100. 1988.

13.C. Lee, W. Yang, and R. G. Parr. "Development of the Colle-Salvetti correlation-energy formula into a functional of the electron density." Phys. Rev. B. v37 p785-789. 1988.

14.S. Goedecker, M. Teter, and J. Hutter. "Separable dual-space Gaussian pseudopotentials." Phys. Rev. B. v54 p1703-1710. 1996.

15.C. Hartwigsen, S. Goedecker, and J. Hutter. "Relativistic separable dual-space Gaussian pseudopotentials from H to Rn.” Phys. Rev. B. v58 p3641-3662. 1998.

16.M. Krack. "Pseudopotentials for $\mathrm{H}$ to $\mathrm{Kr}$ optimized for gradient-corrected exchangecorrelation functionals.” Theor. Chem. Acc. v114 p145-152. 2005.

17.S. Grimme. "Semiempirical GGA-type density functional constructed with a long-range dispersion correction." J. Comp. Chem. v27 n15 p1787-1799. 2006. 
18.S. Grimme, J. Antony, S. Ehrlich and H. Krieg. "A consistent and accurate ab initio parametrization of density functional dispersion correction (DFT-D) for the 94 elements H-Pu." J. Chem. Phys. v132 p154104. 2010.

19.B.T. Kelly. The Physics of Graphite. Applied Science Publishers. New Jersey. 1981. 20.I. Agnarsson, M. Kunter, and T. A. Blackledge. "Bioprospecting finds the toughest biological material: Extraordinary silk from a giant riverine orb spider." PLoS ONE v5 n9 e11234. 2010. 21.K. K. Koski, P. Akhenblit, K. McKiernan, and J. L. Yarger. "Non-invasive determination of the complete elastic moduli of spider silks." Nature Mat. v12 p262-267. 2013.

22.K. Koziol, J. Vilatela, A. Moisala, M. Motta, P. Cunniff, M. Sennett, and A. Windle. "High performance carbon nanotube fiber." Science. v318. p1892-1895. 2007.

23. W. D. Callister, Jr. Materials Science and Engineering. 3rd Edition. New York: Wiley and Sons. 1994.

24. S. J. Deteresa, S. R. Allen, R. J. Farris, and R. S. Porter. "Compressive and torsional behaviour of Kevlar 49 fibre." J. Mat. Sci. v19 p57-72. 1984.

25. M. Cheng, W. Chen and T. Weerasooria. "Mechanical properties of Kevlar KM2 single fiber." J. Eng. Mat. Tech. v127 p197-203. 2005.

26. J. Lim, J. Q. Zheng, K. Masters, and W. W. Chen. "Effects of gage length, loading rates, and damage on the strength of PPTA fibers." Int. J. Impact Eng. v38 p219-227. 2011.

27. R. Grantab, V. B. Shenoy, and R. S. Ruoff. "Anomalous strength characteristics of tilt grain boundaries in graphene." Science. v330 p946-948. 2010.

28. Z. Song, V. I. Artyukhov, B. I. Yakobson, and Z. Xu. "Pseudo Hall-Petch strength reduction in polycrystalline graphene." NanoLetters. v13 p1829-1833. 2013. 
29. A. Cao and J. Qu. Atomistic simulation study of brittle failure in nanocrystalline graphene under uniaxial tension. Appl. Phys. Lett. v102 p071902. 2013.

30. A. Zandiatashbar, G.-H. Lee, S. J. An, S. Lee, N. Mathew, M. Terrones, T. Hayashi, C. R. Picu, J. Hone and N. Koratkar. "Effect of defects on the intrinsic strength and stiffness of graphene." Nature Comm. v5 n3186. DOI: 10.1038/ncomms4186. 2014.

31. P. Zhang, L. Ma, F. Fan, Z. Zeng, C. Peng, P. E. Loya, Z. Liu, Y. Gong, J. Zhang, X. Zhang, P. M. Ajayan, T. Zhu, and J. Lou. "Fracture toughness of graphene." Nature Comm. v5 n3782. DOI: 10.1038/ncomms4782. 2014.

32. Y. Hwangbo, C.-K. Lee, S.-M. Kim, J.-H. Kim, K.-S. Kim, B. Jang, H.-J. Lee, S.-K. Lee, S.S. Kim, J.-H. Ahn, and S.-M. Lee. " Fracture characteristics of monolayer CVD-graphene." Scientific Reports. v4 n4439 p1-9. 2014.

33. G. Nilakantan, A. A. Obaid, M. Keefe, and J. W. Gillespie Jr. "Experimental evaluation and statistical characterization of the strength and strain energy density distribution of Kevlar KM2 yarns: exploring length-scale and weaving effects." J. Comp Mat. v45 n17 p1749-1769. 2010. 34. J. B. Mayo, E. D. Wetzel, M. V. Hosur, and S. Jeelani. "Stab and puncture characterization of thermoplastic-impregnated aramid fabrics." Int. J. Impact Eng. v36 p1095-1105. 2009.

35. D. Roylance, A. Wilde, and G. Tocci. "Ballistic impact of textile structures." Textile Research Journal. v43 n1 p34-41. 1973.

36. J. Sakamoto, J. van Heijst, O. Lukin, and A. D. Schluter. "Two-dimensional polymers: Just a dream of synthetic chemists?" Angew. Chem. Int. Ed. v48 p1030-1069. 2009.

37. P. Kissel, R. Erni, W. B. Schweizer, M. D. Rossell, B. T. King, T. Bauer, S. Götzinger, A. D. Schlüter, and J. Sakamoto. "A two-dimensional polymer prepared by organic synthesis." Nature Chem. v4 p287-291. 2012. 
38. P. Angelova, H. Vieker, N.-E. Weber, D. Matei, O. Reimer, I. Meier, S. Kurasch, J.

Biskupek, D. Lorbach, K. Wunderlich, L. Chen, A. Terfort, M. Klapper, K. Müllen, U. Kaiser, A. Gölzhäuser, and A. Turchanin. "A universal scheme to convert aromatic molecular monolayers into functional carbon nanomembranes." ACS Nano. v7 n8 p6489-6497. 2013. 39. S. W. Cranford and M. J. Buehler. "Mechanical properties of graphyne." Carbon. v49 p4111-4121. 2011.

40. M. Topsakal, S. Cahangirov, and S. Ciraci. "The response of mechanical and electronic properties of graphane to the elastic strain." Appl. Phys. Lett. v96 p091912. 2010.

41. J. Zhou, Q. Wang, Q. Sun, X. S. Chen, Y. Kawazoe, and P. Jena. "Ferromagnetism in semihydrogenated graphene sheet." NanoLetters. v9 n11 p3867-3870. 2009.

42. Y. Li, D. Datta, Z. Li, and V. B. Shenoy. "Mechanical properties of hydrogen functionalized graphene allotropes." Comp. Mat. Sci. v83 p212-216. 2014.

43.M. J. Kory, M. Worle, T. Weber, P. Payamyar, S. W. van de Poll, J. Dschemuchadse, N. Trapp, and A. D. Schluter. "Gram-scale synthesis of two-dimensional polymer crystals and their structure anlaysis by X-ray diffraction." Nature Chemistry. v6 p779-784. 2014.

44.P. Kissel, D. J. Murray, W. J. Wulftange, V. J. Catalano, and B. T. King. Nature Chemistry. v6 p774-778. 2014.

45. Q. Peng, W. Ji, and S. De. "Mechanical properties of the hexagonal boron nitride monolayer: Ab initio study." Comp. Mat. Sci. v56 n11-17. 2012.

46. R. C. Cooper, C. Lee, C. A. Marianetti, X. Wei, J. Hone, and J. W. Kysar. "Nonlinear elastic behavior of two-dimensional molybdenum disulfide" Phys. Rev. B. v87 n035423. 2013. 
Wetzel et al., "A Theoretical Consideration of the Ballistic Response of Continuous Graphene Membranes"

\section{Tables}

Table 1. Comparison of experimental measurement [1] and computational simulations [8 - 10] of the linear and non-linear elastic properties of graphene. Elastic constants for Liu et al. are based on a fit of Eqn. (10) to their published data points.

\begin{tabular}{cccccccc}
\hline \hline Source & $\begin{array}{c}\boldsymbol{E}_{\boldsymbol{m}} \\
(\mathrm{N} / \mathrm{m})\end{array}$ & $\begin{array}{c}\boldsymbol{D}_{\boldsymbol{m}} \\
(\mathrm{N} / \mathrm{m})\end{array}$ & $\begin{array}{c}\boldsymbol{\varepsilon}_{\boldsymbol{m}} \\
(\%)\end{array}$ & $\begin{array}{c}\boldsymbol{\sigma}_{\boldsymbol{m}} \\
(\mathrm{N} / \mathrm{m})\end{array}$ & $\begin{array}{c}\boldsymbol{c}_{\boldsymbol{m}} \\
(\mathrm{km} / \mathrm{s})\end{array}$ & $\begin{array}{c}\boldsymbol{U}_{\boldsymbol{m}} \\
\left(\mathrm{J} / \mathrm{m}^{2}\right)\end{array}$ & $\begin{array}{c}\overline{\boldsymbol{U}}_{\boldsymbol{m}} \\
(\mathrm{J} / \mathrm{g})\end{array}$ \\
\hline$[1]($ Exp.) & 340 & -690 & 25 & 41.9 & 21.4 & 6.88 & 9270 \\
{$[8]$ (DFT) } & 312 & -583 & 27 & 41.7 & 20.5 & 7.44 & 10000 \\
[9] (DFT) & 320 & -685 & 23 & 37.4 & 20.8 & 5.82 & 7840 \\
{$[9](\mathrm{MD})$} & 320 & -615 & 26 & 41.6 & 20.8 & 7.22 & 9730 \\
{$[10]$ (DFT) } & 341 & -771 & 22 & 37.7 & 21.4 & 5.56 & 7490 \\
this study (DFT) & 340 & -805 & 21 & 35.8 & 21.4 & 5.04 & 6790 \\
\hline Average & 329 & -691 & 24 & 39.1 & 21.0 & 6.19 & 8350 \\
\hline \hline
\end{tabular}


Table 2. Comparison of expected ballistic performance of various materials. Materials above the dashed line are textiles, with mechanical properties for fibers / yarns / tows reported. Materials below the dashed line are isotropic membranes. $V_{50}$ ratios in the last column are calculated by normalizing each material's $\Lambda$ by the $\Lambda$ for Kevlar, and multiplying by $\sqrt{2}$ for the isotropic membranes. For most materials, strain energy density is estimated from strength and strain-to-failure data by assuming linear elastic behavior. For the CNT yarn, strain energy is directly reported by the source. Graphene wave speed and strain energy density are from Table 1, with three-dimensional stress and modulus values calculated for comparison purposes by assuming a graphitic layer spacing of $0.335 \mathrm{~nm}$ [19] Sub-graphene is treated as a linear elastic isotropic membrane with a modulus equal to that of graphene, and a strain to failure of $3.3 \%$.

\begin{tabular}{|c|c|c|c|c|c|c|c|c|c|}
\hline Material & Source & $\begin{array}{c}\text { Density } \\
(\mathrm{g} / \mathrm{cc})\end{array}$ & $\begin{array}{c}\text { Young's } \\
\text { modulus } \\
(\mathrm{GPa})\end{array}$ & $\begin{array}{c}\text { Tensile } \\
\text { strength } \\
(\mathrm{GPa})\end{array}$ & $\begin{array}{c}\text { Strain to } \\
\text { failure } \\
(\%)\end{array}$ & $\begin{array}{l}\text { Wave } \\
\text { speed } \\
(\mathrm{km} / \mathrm{s})\end{array}$ & $\begin{array}{c}\text { Strain energy } \\
\text { density } \\
(\mathrm{J} / \mathrm{g})\end{array}$ & $\begin{array}{c}\Lambda_{y} \text { or } \\
\Lambda_{m} \\
(\mathrm{~m} / \mathrm{s})\end{array}$ & $\begin{array}{c}\mathbf{V}_{\mathbf{5 0}} / \\
\mathbf{V}_{\mathbf{5 0}, \text { Kevlar }} \\
(\mathrm{m} / \mathrm{s})\end{array}$ \\
\hline Kevlar 129 (840d aramid yarn) & $\mathrm{a}$ & 1.44 & 96 & 3.38 & 3.3 & 8.2 & 38.7 & 681 & 1.00 \\
\hline Dyneema SK-76 (400d UHMWPE yarn) & a & 0.98 & 88 & 2.62 & 3.6 & 9.5 & 48.1 & 770 & 1.13 \\
\hline Zylon ( 500d PBO yarn) & a & 1.54 & 180 & 5.8 & 3.5 & 10.8 & 65.9 & 893 & 1.31 \\
\hline Nylon (800d polyamide yarn) & a & 1.2 & 5 & 1 & 20 & 2.0 & 83.3 & 554 & 0.81 \\
\hline carbon fiber (7200d PAN-based tow) & a & 1.8 & 234 & 4.83 & 2 & 11.4 & 26.8 & 674 & 0.99 \\
\hline spider silk (Caerostris darwini) & {$[20,21]$} & 1.3 & 11.5 & 1.65 & 50 & 3.0 & 317 & 981 & 1.44 \\
\hline CNT yarn & [22] & 0.9 & 357 & 8.8 & 2.5 & 19.9 & 121 & 1340 & 1.97 \\
\hline aluminum alloy 5083 & $\mathrm{~b}$ & 2.66 & 70.3 & 0.317 & 16 & 5.1 & 9.5 & 366 & 0.76 \\
\hline titanium alloy Ti-6-4 & b & 4.43 & 114 & 1.86 & 14 & 5.1 & 29.4 & 530 & 1.10 \\
\hline AISI 4340 steel, oil-quenched & $\mathrm{b}$ & 7.85 & 196 & 1.86 & 12 & 5.0 & 14.2 & 414 & 0.86 \\
\hline polycarbonate & [23] & 1.2 & 2.38 & 0.066 & 110 & 1.4 & 30.3 & 349 & 0.72 \\
\hline graphene & Table 1 & 2.21 & 981 & 117 & 24 & 21.0 & 8350 & 5600 & 11.6 \\
\hline sub-graphene & & 2.21 & 981 & 32 & 3.3 & 21.0 & 241 & 1720 & 3.57 \\
\hline
\end{tabular}

${ }^{\mathrm{a}}$ manufacturer data ${ }^{\mathrm{b}}$ asm.matweb.com 


\section{Figures}

(a)
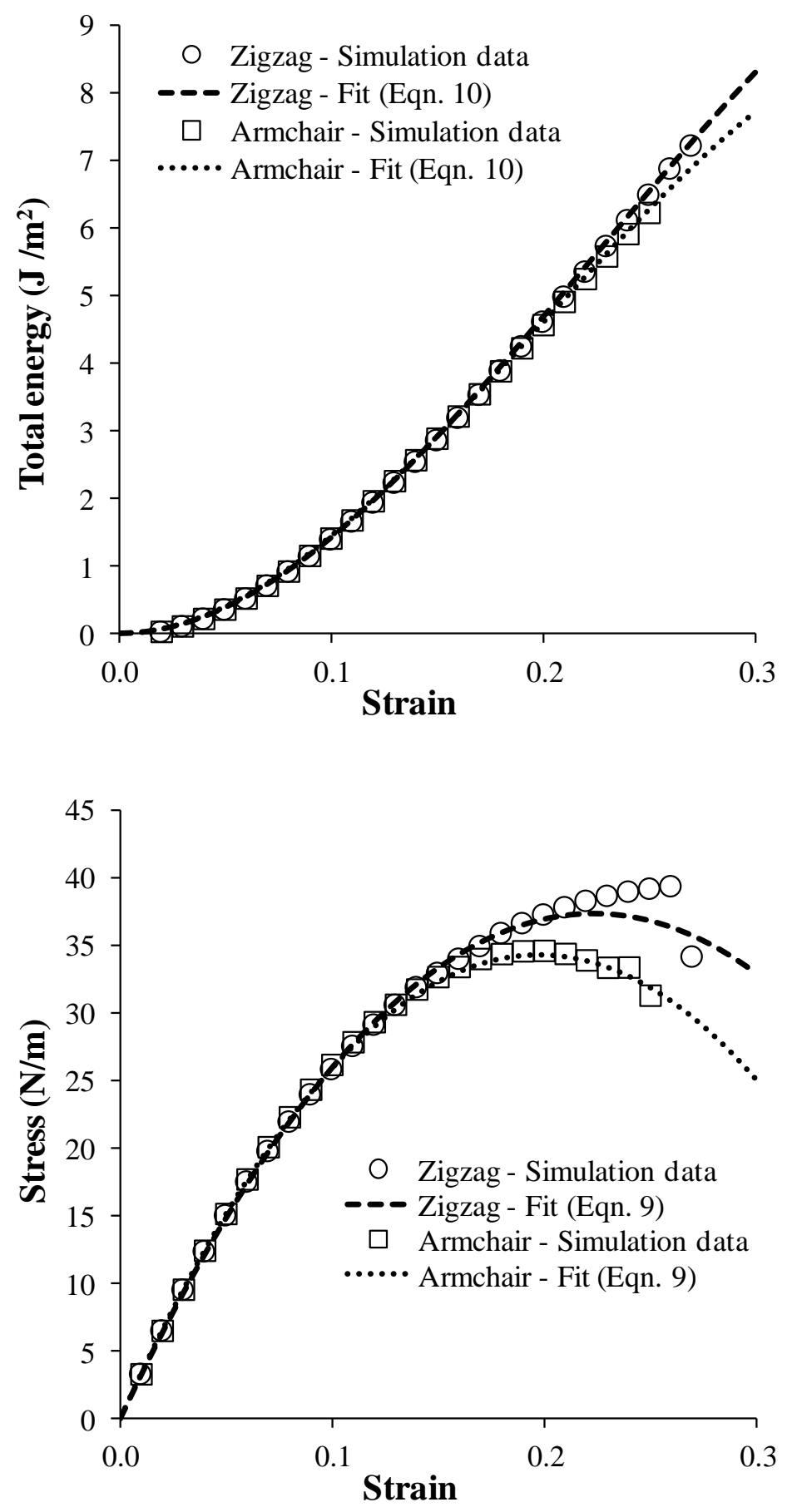

(b)

Figure 1: Computational predictions of (a) lattice energy versus strain and (b) two-dimensional stress versus strain for graphene under uniaxial extension along zigzag and armchair directions.

For clarity, every-other data point from the simulation data set is shown. 


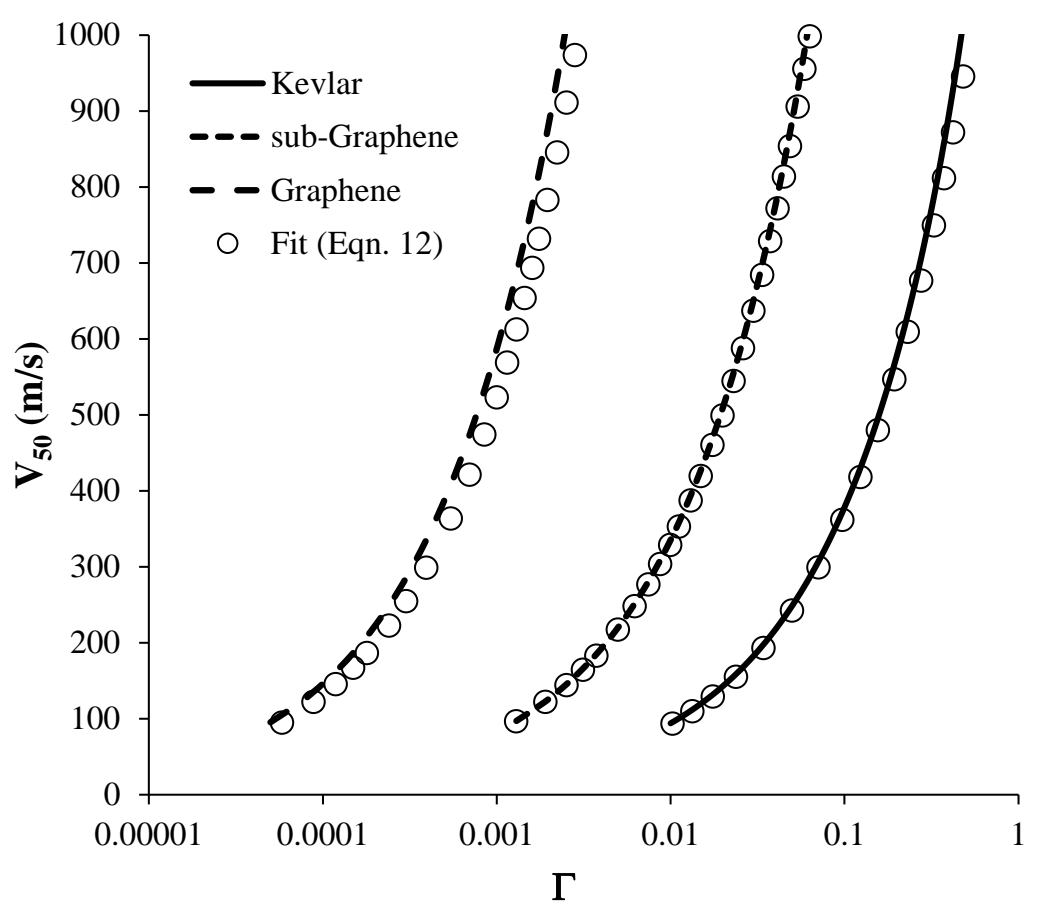

(a)

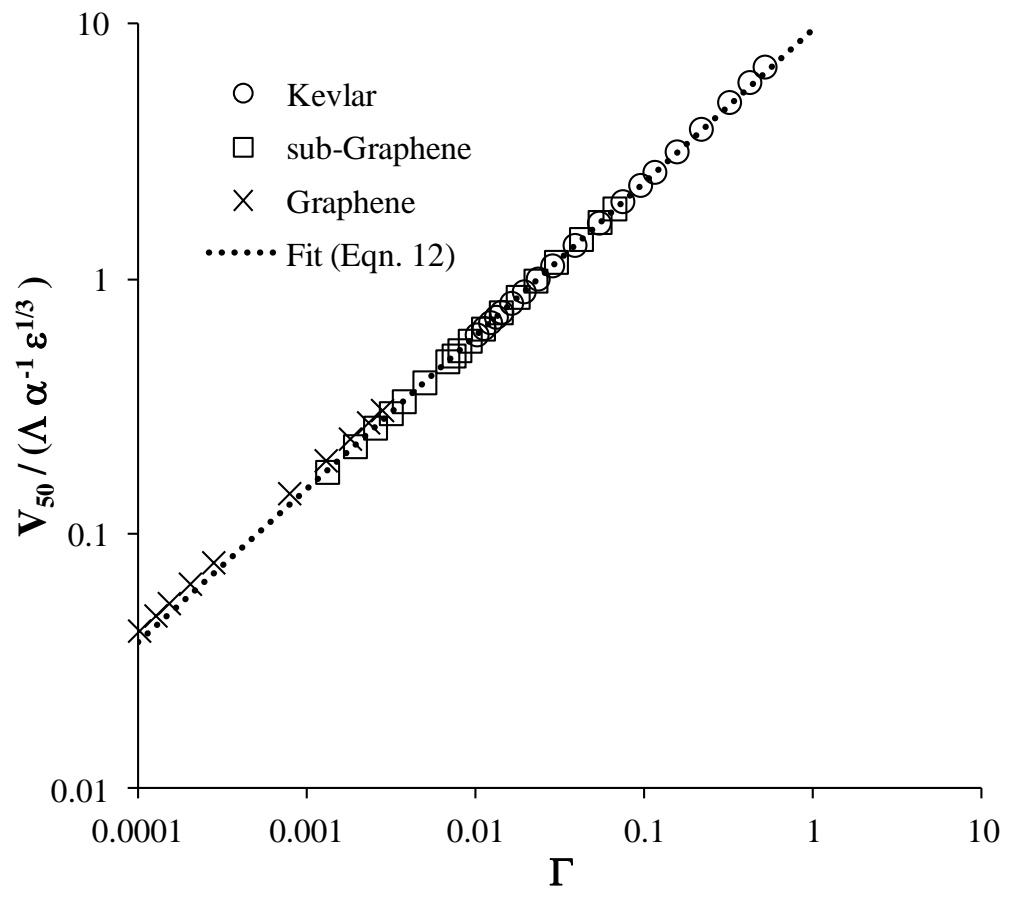

Figure 2. (a) Analytical (Eqns. 5 and 7) and empirical (Eqn. 12) relationships between $V_{50}$ and $\Gamma$ for Kevlar, sub-graphene, and graphene. (b) Scaled $V_{50}$ data as a function of $\Gamma$. 